\title{
COMPRENSIONES EMPRESARIALES DE LA RELACIÓN CON COMUNIDADES MAPUCHE: ALTERIDADES EN TENSIÓN
}

\author{
HOW BUSINESS UNDERSTANDS THE RELATIONSHIP WITH \\ MAPUCHE COMMUNITIES: ALTERITIES IN TENSION
}

\section{NOELIA CARRASCO HENRÍQUEZ}

\section{RESUMEN}

El artículo presenta el análisis de un conjunto de datos recogidos mediante un prolongado proceso de investigación antropológica que abordó las relaciones entre empresas forestales y comunidades mapuche en la Región de La Araucanía. Este artículo se focaliza en el modo en que las empresas forestales, a través de los agentes entrevistados, conciben sus vinculaciones con las comunidades mapuche. Entre los principales resultados obtenidos se descubren orientaciones del capitalismo transnacional en la gestión de las relaciones con las comunidades mapuche, incidentes en las construcciones de la alteridad que dinamizan los cotidianos del territorio.

Palabras clave: Alteridad, empresas forestales, comunidades mapuche, desarrollo económico.

\section{ABSTRACT}

This article presents the analysis of set of data collected through a long process of anthropological research on the relationship between forest companies and Mapuche communities in the Araucanía Region. This analysis focuses on how the forest industry, through the agents interviewed, conceive their relationships with Mapuche communities. Among the main results are orientations of transnational capitalism in the man-

* Dra. en Antropología Social y Cultural, Facultad de Ciencias Sociales, Universidad de Concepción. Concepción, Chile. Correo electrónico: noeliacarrasco@udec.cl 
agement of relations with the Mapuche communities, incidents in the constructions of otherness that invigorate the daily lives of the territory.

Keywords: Otherness, mapuche communities, forest companies, economic development.

Recibido: 08.05.14. Aceptado: 29.03.18.

\section{INTRODUCCIÓN}

L

A HISTORIA DE LAS relaciones entre empresas forestales y comunidades mapuche en Chile se identifica preliminarmente a través de lo que los medios de comunicación han denominado "el conflicto mapuche", usualmente asociado y agravado con referencias al terrorismo y a los enfrentamientos marcados por la violencia. Desde dicha perspectiva, ha sido posible conocer las tensiones, las críticas recíprocas y más aun, la violencia y la criminalización directamente vinculada con el desarrollo de la segunda industria con mayores ingresos en el país, la industria forestal. No obstante, ésta es sólo la cara más superficial de la moneda, pues muestra sólo parte de las evidencias de un proceso histórico, social y cultural mucho más complejo, dejando fuera otros aspectos determinantes para comprenderlo a cabalidad. Entre estos aspectos destacamos a los procesos de acercamiento entre empresas y comunidades, y a las características de las culturas empresariales que definen a la industria forestal presente en el territorio.

El proyecto Fondecyt $11080196^{1}$ abordó, a través de tres casos, las características de las relaciones entre empresas forestales y comunidades indígenas en la Región de La Araucanía, Chile. Estos resultados fueron luego profundizados con los desarrollos de los proyectos Fondecyt $11507770^{2}$ y Anillo PIA SOC $1404^{3}$, los que permitieron ampliar el análisis a la Provincia de Arauco. Los casos implicaban cada uno a una empresa forestal específi-

Proyecto Fondecyt 11080196 denominado "Estudio comparativo de las relaciones entre empresas forestales y comunidades mapuche en la Región de La Araucanía. Discusiones desde la relación entre antropología y desarrollo", fue ejecutado entre noviembre de 2008 y noviembre de 2010.

${ }^{2}$ Proyecto Fondecyt 1150770 denominado "Imaginarios del desarrollo sustentable y ecología política del territorio: conflictos socioambientales y disputas en territorios forestales costeros del Biobío y el Maule".

${ }^{3}$ Proyecto Anillo PIA SOC 1404 denominado "Dinámicas naturales, espaciales y socio-culturales: perspectivas sobre los conflictos socio-ambientales en territorios forestales de Chile, 19752014". 
ca, y a diversas comunidades mapuche con las cuales estas empresas se vinculan. Los criterios antropológicos que permitieron ilustrar y comparar el posicionamiento de las empresas con respecto a las comunidades mapuche han sido: 1. El procedimiento o la modalidad de relación establecida; 2. La definición de los actores mapuche elaborada en la empresa y puesta de manifiesto a través de los planteamientos de los agentes empresariales a través de las entrevistas realizadas; 3 . Los resultados esperados de la relación, entendidos también como el objetivo que cada empresa elabora para fundamentar su interés y decisión de establecer relaciones con las comunidades mapuche. En este último objetivo, se reconoce el sentido sociocultural asignado al otro, en este caso, la visión que los agentes empresariales poseen de los mapuche y que se ve complejizada con los lineamientos que el manejo forestal internacional promueve en materia de derechos indígenas.

A partir de lo anterior, se concluye que existe un horizonte de fines y sentidos compartido por las diferentes empresas forestales estudiadas. No obstante, el análisis comparativo permite identificar diferencias en las rutas utilizadas por cada empresa, basadas en diferencias en los modos de comprender el proceso relacional que han de asumir con el mundo mapuche.

\section{LA CUESTIÓN DE LAS RELACIONES ENTRE EMPRESAS FORESTALES Y COMUNIDADES MAPUCHE}

Los procedimientos o modalidades de relación implementada por cada empresa forestal respecto a las comunidades mapuche, ha sido un objeto de estudio obligado para la comprensión crítica de los procesos territoriales de las últimas décadas en Chile. Siguiendo a Roca (2001), para su abordaje sería insuficiente aplicar únicamente una perspectiva culturalista de la empresa, propia de las ciencias de la gestión. Se hace necesario entonces un tratamiento acucioso de la idea misma de cultura, diferenciado del planteamiento organizacional (de acuerdo al cual se permite comprender un medio identificando aspectos compartidos, estables y estabilizadores, para fines de poder controlarlo mejor). Debemos partir de la consideración de la categoría de cultura empresarial como una categoría heterogénea, pues los datos nos demuestran que si bien existen aspectos comunes entre las distintas empresas, también existen importantes diferencias entre ellas y en el interior de cada una, particularmente en su manera de acercarse y posicionarse con respecto a las comunidades mapuche. De esta manera, adquiere pertinencia una mirada en que la cultura no es una entidad rei- 
ficada ni tampoco un recurso explicativo esencializado de los hechos y la conducta, sino un sistema de interacción de códigos y símbolos asignados, con sentido histórico y presente en las dimensiones formales e informales de la organización. Desde esta mirada a la cultura en empresas transnacionales que interactúan con pueblos indígenas, se abre la oportunidad para abordar la dimensión de la alteridad comprendida de acuerdo a Bhabha (2002), a partir de una definición de la diferencia que “... no debe ser leída apresuradamente como el reflejo de rasgos étnicos o culturales ya dados en las tablas fijas de la tradición. La articulación social de la diferencia... es una compleja negociación en marcha..." (18-19). Desde este marco conceptual inicial, podemos situar con mayor holgura la indagación en torno a las modalidades de acción de las empresas y a las notables variaciones que dichas modalidades han tenido en los últimos años (Carrasco, 2012).

El análisis de los datos permitió identificar diversas modalidades y criterios construidos desde las empresas, signadas como Caso 1, Caso 2 y Caso 3 , los cuales son descritos en el siguiente cuadro:

Cuadro 1. Procedimientos, Identidad Asignada y Resultados Esperados de la relación con las comunidades mapuche.

\begin{tabular}{|c|c|c|c|}
\hline Criterio & Caso 1 & Caso 2 & Caso 3 \\
\hline $\begin{array}{l}\text { Procedimiento } \\
\text { o modalidad } \\
\text { de relación } \\
\text { implementada }\end{array}$ & $\begin{array}{l}\text {-MOFIN } \\
\text {-Forestación asociada } \\
\text {-Apoyo a iniciativas } \\
\text { productivas y } \\
\text { comerciales mapuche }\end{array}$ & $\begin{array}{l}\text {-Programa de } \\
\text { Educación Ambiental } \\
\text {-Diálogos directos } \\
\text {-Forestación asociada } \\
\text {-Apoyo a iniciativas } \\
\text { productivas y } \\
\text { comerciales mapuche }\end{array}$ & $\begin{array}{l}\text {-Programa de } \\
\text { Forestación Mapuche: } \\
\text { Convenios de } \\
\text { Forestación } \\
\text {-Capacitación a } \\
\text { trabajadores mapuche } \\
\text { en faenas forestales }\end{array}$ \\
\hline $\begin{array}{l}\text { Definición de } \\
\text { actor mapuche } \\
\text { / Identidad } \\
\text { Asignada }\end{array}$ & $\begin{array}{l}\text {-Comunidad - } \\
\text { individuo }\end{array}$ & -Comunidad & $\begin{array}{l}\text {-Propietario individual } \\
\text {-Propietario } \\
\text { comunitario }\end{array}$ \\
\hline $\begin{array}{l}\text { Resultado } \\
\text { Esperado de } \\
\text { la relación }\end{array}$ & $\begin{array}{l}\text {-Desarrollo económico } \\
\text { de las comunidades } \\
\text { mapuche, } \\
\text { desaparición de la } \\
\text { pobreza en el entorno } \\
\text { de la empresa }\end{array}$ & $\begin{array}{l}\text {-Conservación del } \\
\text { medio ambiente, } \\
\text { sustentabilidad } \\
\text { social del negocio } \\
\text { forestal. }\end{array}$ & $\begin{array}{l}\text {-Promoción del } \\
\text { negocio de la } \\
\text { producción de } \\
\text { madera }\end{array}$ \\
\hline
\end{tabular}

Los antecedentes sintetizados en el Cuadro 1 muestran que dos de las empresas comparten modalidades de relación, dentro de lo que podemos 
reconocer como sus principios y sus prácticas. En efecto, los casos 1 y 2 coinciden en plantearse ante las comunidades mapuche desde una visión estratégica, es decir, reconociendo que "el tema mapuche" es fundamental para el adecuado desarrollo del negocio. Desde esta posición común, ambas empresas destinan recursos para el diseño de mecanismos que garanticen tanto una "buena relación" con las comunidades, como también dar cumplimiento a sus fines relativos a la vinculación con el entorno y a la sustentabilidad, respectivamente. Este último aspecto es particularmente importante, puesto que puede permitirnos explicar algunas diferencias en los procesos que cada una de estas empresas han experimentado en la construcción de sus acercamientos a las comunidades mapuche.

En el caso 1, se puede documentar que efectivamente esta empresa habría sido pionera en el intento por establecer una modalidad de acercamiento particularmente innovadora, desde una gran empresa forestal hacia las comunidades. Evidentemente el programa MOFIN ${ }^{4}$, integrado a las estrategias de organización industrial, constituyó en el año 2008 un hito tanto dentro de la empresa como fuera de ella, considerando a su vez el efecto que supuso para otras empresas forestales que compartían la preocupación por cómo enfrentar el tema de su relación con las comunidades mapuche 5 . La importancia de este hito es que aun cuando no implicase transformaciones radicales en los órdenes ideológicos o estructurales de la empresa como tal, expresó la posibilidad de que éstas replanteasen su modalidad de relación con las comunidades mapuche. En lo concreto, antes del MOFIN, era altamente improbable oír a ningún agente empresarial hablar de "la cultura mapuche" y su protección, ni tampoco poner de manifiesto una preocupación por el desarrollo económico de las comunidades, en el sentido que lo ilustra la siguiente cita de un profesional de la empresa Caso 1:

Nosotros pensamos en la costa y esta propuesta ya es ver potencialidades de la zona que permitan que las comunidades mapuches tengan un desarrollo real y nosotros apoyar las iniciativas que ellos puedan tener,

\footnotetext{
${ }^{4}$ Modelo Forestal Intercultural.

${ }^{5}$ La implementación inicial que la empresa que protagoniza el caso 1 hizo del Modelo Forestal Intercultural (MOFIN) fue extensamente documentado por un equipo interdisciplinario de investigadores de la Universidad Católica de Temuco, en el periodo julio 2008 - julio 2009. Para más antecedentes, ver "Informe Final del Proyecto Diversidad Cultural y Desarrollo en la Región de la Araucanía. Seguimiento interdisciplinario a la implementación del MOFIN en los procesos de desarrollo de dos comunidades mapuche de la comuna de Chol-Chol", Samaniego, M. et al. Documento en propiedad de los investigadores, de los dirigentes de las comunidades y de la empresa.
} 
porque si a ellos les va bien a nosotros nos va bien también y en la zona de Tirua y el lago Lleu lleu y la costa Lafkenche es el turismo y eso es un gran potencial para hacer algo en lo cual la empresa puede ayudar y que las comunidades puedan aplicar todo lo que es su conocimiento cultural. No turismo por hacer turismo sino que un turismo distinto.

(J. N. 08.01.09)

Esta posición de la empresa caso 1, permite situar allí a lo que se considera como el hito inicial de un proceso de redefinición en las relaciones entre las empresas forestales y las comunidades mapuche, que no puede ser sindicado como un proceso de integración convencional. Pareciera ser que este hito inicia la instalación de una modalidad de relación que si bien no es única y exclusiva dentro de la gama de posibilidades, sí da cuenta de la adhesión de las empresas a un supuesto cultural hegemónico, a saber: que las comunidades aspiran a un desarrollo económico que pase por su integración a los mercados y a la economía formal. La reproducción de este supuesto dependería de que las comunidades asuman que las empresas forestales "llegaron para quedarse" y por lo tanto, han de aceptar el establecimiento de vínculos que desde su punto de vista también puede ser visto como estratégico. En este sentido, se ha constatado que para algunas comunidades y organizaciones mapuche la vinculación con las empresas forestales puede ser vista como una estrategia para la obtención de sus fines declarados en sus proyectos de desarrollo, no excluyente e integrada en una posición que puede seguir siendo reivindicativa de derechos y de recuperación de recursos como la tierra ${ }^{6}$. Ante esto, algunas empresas han asumido la necesidad de replantear su acercamiento al mundo indígena, diseñando e implementando aproximaciones directas, asentadas a su vez en la reformulación de algunos aspectos de sus discursos corporativos ${ }^{7}$.

Ciertamente, se trata de una coyuntura ideológica y empírica bien particular. Los discursos de las partes siguen siendo marcadamente diferenciables, no obstante ambos reconocen la importancia estratégica y el carácter instrumental que puede llegar a tener la relación, y le asignan un sentido dentro del cumplimiento de sus fines independientes y particulares.

6 Asociación que agrupa a comunidades mapuche, integrada a la agrupación empresarial CorpAraucanía, bajo la opción de integrarse al esquema del desarrollo económico productivo de la gran empresa, demandándoles capacitación y también apoyo en sus iniciativas productivas.

${ }^{7}$ Expresión de estos replanteamientos en empresas como el caso 1 se encuentran en la inclusión del MOFIN a partir de una evaluación crítica del modelo de relación promovido por los programas de Buena Vecindad, de acuerdo a los cuales, la empresa únicamente actúa de modo unilateral (desde ella hacia las comunidades), sin atender ni responder a lo que las comunidades están planteando, a diferencia de lo que sucedería bajo la modalidad MOFIN. 
El caso 2 da cuenta de una planificación de las relaciones que la empresa construye con actores y comunidades mapuche, a partir de directrices tales como la sustentabilidad y el desarrollo económico. En este caso, se observa que se privilegia el vínculo formal a través de figuras representativas de la comunidad, por sobre los apoyos individuales o los reconocimientos a personas específicas. Al igual que en el caso 1, se evidencia también que la empresa hace un uso expansivo del supuesto del desarrollo económico de las comunidades mapuche, reafirmando que el vínculo tiene un sentido estratégico y ético en el apoyo que como empresa se pueda prestar a las iniciativas productivas y comerciales que puedan emprender las comunidades. Destaca especialmente en este caso, el énfasis puesto a la cuestión de la sustentabilidad y la conservación del medio ambiente en tanto contenido que justifica el establecimiento de relaciones con las comunidades mapuche. Este aspecto es relevante, dado que amplía el marco argumentativo del desarrollo económico y asocia al comportamiento empresarial forestal con el modelo de manejo forestal sustentable vigente en los territorios a través de los procesos de certificación forestal internacional bajo los parámetros del Forest Stewardship Council (FSC) y Programme for the Endorsement of Forest Certification (PEFC) homologado en Chile por CERTFOR (Certificación Forestal). El escenario creado por estos instrumentos del mercado internacional ha contribuido notablemente a la promoción de la renovación de los vínculos entre las empresas forestales y comunidades mapuche, pues presionan especialmente a que las empresas presten atención a contenidos tales como los derechos indígenas, el desarrollo local y la conservación de la biodiversidad (Carrasco, 2015).

El caso 3 da cuenta de un posicionamiento que se distingue de los anteriores por su carácter marcadamente etnocentrado y reducido en el discurso y la práctica a la integración de propietarios y comunidades mapuche al negocio forestal. En concordancia con ello, los agentes empresariales reconocen al mundo mapuche a través de la figura individual, apta para el establecimiento de acuerdos comerciales en calidad de "socio". A partir de ello, busca proyectar la construcción de una aparente simetría, en que individuos mapuche de un lado y la empresa de otro, se contribuyen mutuamente para obtener beneficios económicos. Esta postura ha sido reconocida en análisis previos como parte de un estilo empresarial irreflexivo y disociado, que refleja la ausencia de comprensión por parte de la empresa del escenario culturalmente diverso en que desarrollan la actividad forestal (Carrasco, 2011). 


\section{ALTERIDADES EN TENSIÓN}

De acuerdo a Todorov (2008), la relación con el otro no se constituye en una sola dimensión. Comprender las relaciones sociales desde una perspectiva de alteridad exige reconocer al menos tres planos implicados: el axeológico, el praxeológico y el epistémico.

En el plano axiológico podríamos visualizar los contenidos elaborados a través de los juicios de valor; aquí encontraríamos la sustancia de lo que denominamos predisposiciones o actitudes, las justificaciones valóricas a través de lo que se concibe como bueno o malo, siempre desde la autorreferencialidad. "Las comunidades tienen potencialidades" / "Las empresas siempre van a buscar su conveniencia".

En el plano praxeológico en tanto, identificamos las acciones de acercamiento o alejamiento. Desde esta mirada, podemos vislumbrar las distancias y las brechas que suponen tensión o conciliación. Una tipología básica nos permitiría comprender desde este plano praxeológico tres posibilidades mínimas: identificación con el otro, negación del otro, neutralidad o indiferencia ante el otro. Ciertamente que estas categorías pueden imbricarse o intermediarse por ejemplo, con expresiones de distancia relativa, sobre todo en un escenario tan complejo como el estudiado, en que las empresas representan un orden expansionista que sobrevive y busca coexistir con fuerzas locales heterogéneas (Ribeiro, 2003), y en el que estas fuerzas locales heterogéneas recrean a su vez mecanismos de supervivencia, en el marco de la tensa relación entre concepciones locales y supralocales.

Finalmente, el plano epistémico nos muestra la dimensión del conocimiento/ignorancia del otro, su identidad, su historia, sus circunstancias. Los datos nos permitirán ilustrar que tanto este plano como los otros dos pueden modificarse con el tiempo en virtud de las determinantes históricas y de contexto; de otro modo, no podríamos explicar ni los cambios ni las crisis en las relaciones sociales. Por otra parte, podrá ilustrarse que este plano no tiene necesariamente una relación causal con los dos anteriores, es decir, "el conocimiento no implica el amor ni a la inversa; y ninguno de los dos implica por la identificación con el otro, ni es implicado por ella" (Todorov, 2008, p. 222).

Para examinar más detenidamente estas posiciones determinantes de las cualidades de la alteridad, se analizaron las visiones de actores de empresas sobre las comunidades mapuche, a partir de los resultados arrojados por el software de Análisis Cualitativo Atlas Ti 6.0.

Los resultados que arrojó este análisis nos presenta el posicionamiento 
de la empresa Caso 1, sostenido a partir de una autoevaluación positiva de la relación que se mantiene con las comunidades mapuche, basada en la calidad de la recepción que -desde su punto de vista- ha tenido su acercamiento a éstas. La empresa -través de sus agentes ejecutivos y profesionales- considera estar procediendo adecuadamente. En el plano axiológico entonces, podemos inferir que la empresa asume hacerlo bien, frente a otro que considera bueno y por ende, digno de una relación positiva. Esta posición se refirmaría más aún, con la consideración del otro como "necesitado", comunidades mapuche que requieren el apoyo de externos para "generar desarrollo", y la auto identificación de la empresa como un actor que posee condiciones y recursos para prestar ese apoyo. Desde esta lógica, la empresa justifica la pertinencia de su presencia y de su intencionalidad en el cumplimiento de un rol ante otro diferente, al que considera parte de un sistema económico y social compartido, en un territorio común.

Por otra parte, se evidencia una visión positiva de las comunidades, toda vez que se asume que éstas son portadoras de valores que la empresa comparte. En lo concreto, se rescata que estén organizados, es decir, se aprecia positivamente que cumplan con este valor tan exacerbado en la modernidad, especialmente a través de la presencia de líderes mapuche que cumplan con el requisito de valorar y respetar la presencia empresarial. Allí se manifiesta nuevamente una finalidad para la relación con las comunidades que portan este valor de la organización: reconocerles su organización e integrarla. "No es como yo, pero puede serlo".

Finalmente, se reconoce desconocer la realidad de las comunidades. Cuando los agentes empresariales dicen "no sabemos del tema", y simultáneamente figuran liderando y participando de nuevas fórmulas de relación, muestran que el desconocimiento no constituye en su visión un impedimento para vincularse. Esta idea se reforzaría, al comprender que para la empresa se trata de una estrategia necesaria y en la que se procede de modo adecuado.

En el Caso 2, la empresa organiza su relación con las comunidades mapuche a partir de dos grandes posicionamientos distintos, pero no excluyentes entre sí. Por un lado, se expresa una contundente visión positiva hacia éstas, lo cual fundamentaría que la empresa se relacione con ellas por medio de sus diversas modalidades. Por otro, se mantendría una visión crítica hacia el perfil de algunas comunidades que no responderían a los valores que la empresa reconoce como necesarios e indispensables para una buena relación.

En el plano axeológico, la empresa muestra igualmente una configura- 
ción compuesta, entre la valoración positiva hacia las comunidades con las cuales no ha tenido conflictos y una valoración negativa hacia aquéllas con las cuales sí lo ha tenido. Podemos inferir, entonces, que para esta empresa hay distintos tipos de comunidades y con ello se explicaría por qué ofrece distintas modalidades de relación hacia ellas.

En el plano praxeológico entonces, observamos una dualidad: por un lado hay una voluntad de acercamiento y por otro, existe un acercamiento forzado por los requerimientos del negocio, que le exige a la empresa perseverar en una disposición al diálogo. Estas condiciones se evidencian en procesos que igualmente pueden definirse como de distancia relativa, considerando que la empresa asume el acercamiento pero lo hace distinguiendo entre lo que sería, por un lado, apoyar el desarrollo de las comunidades y por otro, facilitar la resolución de conflictos y evitar obstáculos a las faenas.

En último término, en el plano epistémico, el Caso 2 se destaca por su aproximación institucional en todos los órdenes de relación que impliquen a las comunidades mapuche. A razón de ello, es que todas sus vinculaciones obedecen a las formalidades reconocidas, a la validación de las estructuras, jerarquías y competencias que los actores posean para participar, acordar y decidir lo que corresponda. Este principio de reconocimiento del otro sería parte del carácter conservador que identificamos como propio de esta empresa, y tendría una expresión empírica en la caracterización de las relaciones que efectivamente establece con las comunidades. En virtud tanto del carácter conservador pero también de la presencia concreta de la empresa en las relaciones diversas con las comunidades, epistémicamente la ubicaríamos en la misma categoría que la empresa Caso 1: "desconozco, pero quiero", es decir, hay relación desde los marcos y códigos reconocidos - institucionales, jurídicos - puesto que le proporcionan seguridad y rigor; no podría haberla desde otras coordenadas porque la empresa no maneja otras y no asumirá modalidades de relación que no se ajusten a su comprensión.

A diferencia de lo observado en los otros dos casos estudiados, los agentes del Caso 3 poseen una visión marcadamente cerrada hacia las comunidades. Efectivamente, esta empresa posee una visión articulada en torno a principios rígidamente establecidos, tanto en lo estrictamente económico como también en lo social.

Manifiesta a través de sus agentes una visión positiva de las comunidades mapuche y de la relación que han construido entre ambas partes. No podría ser de otro modo, dado que la relación con las comunidades es la estrategia básica que permite la efectividad del Programa de Forestación que promueve la empresa, exclusivamente dirigido a población mapuche; 
sin que los propietarios mapuche pusiesen a disposición sus tierras para plantar, el programa no podría existir. Ello explica la existencia de una visión de la comunidad mapuche como referentes de sociedad (comercial) para el negocio forestal, sin por ello negarles la condición étnica de ser mapuche. No obstante, los datos ilustran que este último aspecto es sólo una condición que la empresa asume manejar de modo estratégico y exitoso, pues lo que constituye el centro de la relación es el desarrollo de la actividad forestal y, por tanto, la condición de la diferencia cultural no constituye una variable problemática sino estratégica. Lo que sí puede ser un problema es el desconocimiento de la actividad forestal por parte de las comunidades, pues el hecho de que éstas no conozcan sus beneficios les impediría -de acuerdo al punto de vista de la empresa- gozar de estos. Dado lo anterior, el sentido axiológico podría encontrarse en la visualización de las comunidades como un otro poseedor de recursos, útil para la expansión del negocio.

En la dimensión praxiológica entonces, se descubre una proximidad instrumental, pues en el Caso 3 la relación con las comunidades se debe principalmente a la modalidad contractual que exige la estrategia comercial. Si bien la empresa despliega otro tipo de acciones, como por ejemplo la capacitación de personas mapuche en faenas forestales -contribuyendo con ello a la formación de capital humano para el rubro-, todas sus modalidades obedecen al mismo sentido: integrar a los mapuche a la actividad forestal, aproximarlos, familiarizarlos.

Lo anterior permitiría plantear que el plano epistémico de la visión de la empresa hacia las comunidades mapuche se define al considerarlos como un actor diferente, pero lo suficientemente conocido. Es decir, le desconozco en su condición cultural diferente y propia, pero ello no es obstáculo para efectuar el vínculo que se requiere a través de los Convenios de Forestación. Dicho de otro modo, en el planteo epistémico de la empresa no es requisito conocer al otro más allá de su condición de socio comercial.

\section{CONCLUSIONES}

Las categorías que las empresas forestales validan al momento de establecer un vínculo concreto con la población mapuche son básicamente dos: el individuo y la comunidad. En el plano individual podemos identificar que las empresas reconocen a los mapuche en su condición de personas pertenecientes a una etnia o cultura distinta, sobre las cuales recae un interés, dada su condición de propietarios de tierra. En el plano comunitario se observa 
que las empresas reconocen a los mapuche como miembros de organizaciones con las cuales se interactúa a través de sus dirigentes o representantes y también como comunidades que pueden ser reconocidas como tales, dado que igualmente constituyen figura jurídica habilitada para participar de contratos económicos o acuerdos comerciales como los requeridos por los Convenios de Forestación. En esta condición, no es relevante el origen étnico cultural, el cual resulta secundario frente a la condición de "ser propietarios" y por tanto, poder participar de un acuerdo comercial con la empresa.

Por otra parte, las empresas forestales analizadas coincidirían en su aspiración a que la relación con las comunidades cumpla un conjunto de expectativas, entre las cuales identificamos las siguientes: que los mapuche se integren a la economía formal, incentivando la innovación para la articulación de su producción a los mercados; que se avance cualitativa y cuantitativamente en la superación de la pobreza presente en las comunidades; que el negocio forestal cumpla con los requisitos de sustentabilidad social; que el patrimonio productivo de los mapuche sea integrado a la industria forestal y, finalmente, que las comunidades participen en mercados globales, incluyendo en ellos el de la industria forestal.

Todo lo anterior, permite evidenciar la prevalencia de un horizonte de fines compartido por los diferentes casos estudiados. No obstante, el análisis comparativo nos permite identificar matices en las rutas o fórmulas utilizadas por cada empresa, presumiblemente derivadas de las características diferenciables de sus respectivas dinámicas culturales internas, los perfiles de sus profesionales y la orientación de sus formaciones. Estas características proveerían, en cada caso, de distintos modos de comprender el proceso relacional que como empresa han de asumir con el mundo mapuche.

Los casos 1 y 2 no comparten la opción de usar exclusivamente la forestación asociada como estrategia de vinculación. Desde sus puntos de vista, ésta es sólo una modalidad posible y no constituye una respuesta a la debida relación que políticamente estarían obligados a construir con las comunidades. Para estas empresas es posible recrear estrategias de acercamiento, desde una postura en que reconocen que el capitalismo puede ensayar nuevas fórmulas de gestión y administración (Colobrans, 1996). Los nuevos estilos de liderazgo y managment han favorecido que estas empresas se movilicen internamente en un sentido reflexivo, ampliando sus criterios de comprensión de la situación de los mapuche y su incidencia en el negocio forestal. 
No sucede lo mismo con la empresa del caso 3 , que a diferencia de las anteriores, no se identifica en el escenario, no toma distancia para observarse como actor en el territorio, sino que sólo juega un rol económicoproductivo en él. En este caso, no se evidencian signos de revisión de filosofías corporativas sino sólo la expresión permanente de afirmaciones dogmáticas en torno a las ventajas de la producción forestal.

\section{REFERENCIAS}

Bhabha, H. (2002). El lugar de la cultura. Buenos Aires, Argentina: Editorial Manantial.

Carrasco, N. (2011). “Aproximación etnográfica a los estilos socioculturales en la gestión empresarial. Despliegues en la región de la Araucanía”. Sociedad Hoy, 21, 67-77.

. (2012). "Trayectoria de las relaciones entre empresas forestales y comunidades mapuche en Chile. Aportes para la reconstrucción etnográfica del desarrollo económico en contextos interétnicos". Polis, 31, 355-371.

. (2015). "Pueblos indígenas y biodiversidad en el capitalismo contemporáneo". Revista Justicia do Direito, 29(1), 88-107.

Colobrans, J. (1996). "Antropología de la empresa. Culturas corporativas y culturas organizativas". En Prat, J. y Martínez, A. (eds.), Ensayos de antropología culturas. Homenaje a Claudio Esteva-Fabregat (pp. 262-269). Barcelona: Ariel.

Ribeiro, G. (2003). Postimperialismo. Cultura y política en el mundo contemporáneo. Buenos Aires: Gedisa.

Roca, J. (2001). “¿Antropólogos en la empresa?: A propósito de la (mal) llamada cultura de la empresa". Revista Etnográfica, V(I), 69-99. Disponible en: http://ceas.iscte.pt/etnografica/docs/vol_05/N1/Vol_v_N1_69-100.pdf (13 de septiembre de 2010).

Todorov, T. (2008). La conquista de América. El problema del otro. Buenos Aires: Siglo Veintiuno Editores. 